\title{
Association between the urban environment and chronic disease to identify communities at risk
}

\author{
Ken Wei Tan ( $\nabla$ e0145748@u.nus.edu ) \\ National University of Singapore https://orcid.org/0000-0003-3224-4619 \\ Qianyu Yang \\ National University of Singapore \\ Yin Ai Lean \\ National University of Singapore \\ Joel Ruihan Koo \\ National University of Singapore \\ Alex R Cook \\ National University of Singapore \\ Borame L Dickens \\ National University of Singapore
}

\section{Research}

Keywords: Statistical modelling, chronic disease, spatial epidemiology, urbanisation, environmental health

Posted Date: December 6th, 2019

DOl: https://doi.org/10.21203/rs.2.18164/v1

License: (c) (i) This work is licensed under a Creative Commons Attribution 4.0 International License.

Read Full License 


\title{
Association between the urban environment and chronic disease to identify communities at risk
}

\author{
Ken Wei Tan ${ }^{1}$, Qianyu Yang ${ }^{1}$, Yin Ai Lean ${ }^{1}$, Joel Ruihan Koo ${ }^{1}$, Alex R Cook ${ }^{1}$, \\ Borame L Dickens ${ }^{1}$
}

${ }^{1}$ Saw Swee Hock School of Public Health, National University of Singapore and National University Health System, Singapore.

Pre-publication Corresponding Author:

Tan Ken Wei

Saw Swee Hock School of Public Health

National University of Singapore

12 Science Drive 2

Singapore 117549

Email: $\underline{\text { e0145748@u.nus.edu }}$

\section{Post-publication Corresponding Author:}

Borame L Dickens

Saw Swee Hock School of Public Health

National University of Singapore

12 Science Drive 2

Singapore 117549

Email: ephdbsl@nus.edu.sg

Keywords: Statistical modelling, chronic disease, spatial epidemiology, urbanisation, environmental health 


\begin{abstract}
$\underline{\text { Abstract }}$
Background: With increasing urbanisation rates, assessments must be made on the impact of the built environment on the health of populations. As the bulk of healthcare expenditure in developed countries is borne by the elderly through chronic disease management and treatment costs, intervening using the built environment can have lasting population-wide effects.
\end{abstract}

Methods: Using two cohort studies for training and validation, we quantified each individual's local context based on their residential address and derived geographical exposures adapted from the International Physical Activity and the Environment Network guidelines. Bayesian inference was used to develop a regression model that examines the impacts of the geographical exposures and predicts mean body mass index and prevalence of type 2 diabetes mellitus, acute myocardial infarction and stroke by communities.

Results: The distance to the nearest retail outlet was found to be negatively associated with body mass index. Our prediction model shows good accuracy (AUC $>0.75$ ) for predicting type 2 diabetes mellitus, acute myocardial infarction and stroke. National-level maps were generated that predict the health of communities by mean body mass index and overall chronic disease risk.

Conclusions: The predictive model has the ability to predict on a macro scale the overall health of a community. Understanding the geospatial distribution of chronic disease risk allows for evidencebased policymaking with urban-specific interventions that improve overall population health. 


\section{$\underline{\text { Introduction }}$}

Chronic diseases are the main cause of mortality and morbidity in developed countries, and constitute a large proportion of the healthcare burden ${ }^{1}$. The direct medical costs of various chronic diseases have been estimated to be US\$3,200-4,700 for each type II diabetes mellitus (DM) case annually ${ }^{2}$ and US\$14,000 and US\$25,000 for each episode of acute myocardial infarction (AMI) and acute ischaemic stroke respectively ${ }^{3}$. Globally, rising life expectancy has resulted in aging populations in countries such as Japan and Taiwan. From 2006 to 2015, the DM prevalence in Japan increased from $12.3 \%$ to $19.5 \%$ for men and $8.2 \%$ to $9.2 \%$ for women ${ }^{4}$. In Taiwan, the prevalence of multiple chronic conditions increased from $9.6 \%$ to $17.1 \%$ from 2000 to $2010^{5}$. The prevention of chronic diseases is therefore of a priority to mitigate the rising healthcare costs that accompanies societal aging. High body mass index (BMI) is associated with increased chronic disease progression and mortality ${ }^{6}$. In Singapore, an island city state, obesity and DM incidence have been increasing since the 1990s, alongside other chronic complications such as AMI and stroke. For residents aged 18-69 in the 1992 National Health Survey, the proportion of obese and overweight individuals was $5.1 \%$ and $26.2 \%$. By 2017 , these figures surged to $8.7 \%$ and $36.2 \%$ with DM prevalence also increasing to $8.6 \%$ from an estimated $8.3 \%$ in the last National Health Survey conducted in $2010^{7}$. From 2007 to 2016, the annual number of AMI and stroke cases increased from 6,817 to $10,728^{8}$ and 5,578 to $7,413^{9}$, indicative of ongoing lifestyle changes and an ageing population.

Many epidemiological studies explore the relationship between chronic disease and its risk factors, many of which are sociodemographic or behavioural ${ }^{10,11}$. Fewer studies explore the relationship between the built environment, which is particularly relevant for high density cities such as the growing urban centres of Asia, and health outcomes. Greater walkability has previously been shown to be associated with lower obesity and DM risk (odds ratios of $0.83[0.77-0.91]$ and $0.86[0.75-$ $0.99])^{12}$. Lack of access to healthy food options can encourage a poor diet, which is a major risk factor for obesity, cardiac conditions and stroke ${ }^{13}$. In a US study, simulations of increased supermarket density to improve access to healthier food options resulted in a neighbourhood-wide reduction of $0.09(0.02-0.16)$ BMI points ${ }^{14}$. Those living in food deserts reported a hazard ratio of 1.44 (1.06- 
1.95) of developing an AMI ${ }^{15}$. Similarly, those with fast-food restaurants in their neighbourhood were at higher risk of developing stroke (odds ratios of 1.02 for males and 1.03 for females) in a Swedish study with over 4 million individuals ${ }^{16}$. These studies share consistent directions of effect and a plausible mechanism, suggesting that access to food is associated with obesity and various chronic conditions. These conclusions warrant some investigation as to whether the observed pattern holds true for highly urbanised and population dense countries such as Singapore or Hong Kong, which differ from many of the primarily western-centric studies both geographically and behaviourally ${ }^{17}$.

To answer these questions, this paper has two primary objectives. We aimed to quantify the effect of the urban environment on the health of the resident population of Singapore using a Bayesian approach, as well as predict the expected national risk. Using this methodology, we identify communities in Singapore with large chronic disease risk for the purpose of public health intervention planning which can support the national chronic disease management program set up in 2006 to prepare for the increasing healthcare needs of a rapidly aging population ${ }^{18}$. The identification of these high-risk communities allows for both early interventions to prevent chronic disease onset as well as the strategic placement of primary care facilities to service growing healthcare needs of the population. 


\section{$\underline{\text { Methods }}$}

The Singapore Multi-Ethnic Cohort (MEC) study is a closed cohort recruited from 2004 to 2010 that has a total of 14,465 adult individuals with oversampling of ethnic Malays and Indians ${ }^{19}$, Singapore's two main ethnic minorities. The intentional oversampling of ethnic minorities allowed for more accurate risk estimates for DM, AMI and stroke, as well as ethnic-specific BMI distributions. The Community Health Study (CHS) focused on recruiting specifically from two mature estatesQueenstown and Bukit Panjang-with a predominantly older demographic $(\mathrm{n}=7844,3.3 \%$ of total population in the area). Information on established risk factors such as age, ethnicity, gender, smoking status and dwelling type were collected for adjustment in the final model ${ }^{20-22}$. Dwelling type as a covariate together with the individual's postal code was further post-processed to derive house price (Supplementary information 1). These two cohorts were selected to be the training and validation datasets as detailed socio-demographic information and health outcomes of interest were available, and the questionnaires were standardised during both time periods to prevent misclassification of risk factors and outcomes. For this study, a subset of the MEC $(n=10,499)$ and CHS $(n=5,275)$ was selected that had complete data on all risk factors for the training and validation datasets, respectively.

\section{Derivation of exposure explanatory variables}

Our procedure for quantifying the built environment was adapted from the International Physical Activity and Environment Network (IPEN) adult study, in particular their definitions for land use and environmental attributes in an urban setting ${ }^{23,24}$. For each individual in the cohort, we used a 500meter radial buffer based on their residential postal code to define their urban environment; Singapore being a compact city state with high connectivity, there are small discrepancies between radial buffers and network buffers ${ }^{25}$. Data on land use type and facilities are obtained from multiple government agencies, primarily from the Urban Redevelopment Authority ${ }^{26}$, National Parks Board, Land Transport Authority and National Environment Agency. Polygonal maps of land use for residential, retail, civic/institutional, public parks and private recreational purposes were generated, which were further supplemented with the locations of public transport access points and food establishments. IPEN guidelines recommend a few different metrics to quantify the urban environment, of which we 
selected area of a specific land use type within the 500-meter radial buffer and distance to the nearest establishment within a class of premises due to interpretability. IPEN land use types within an individual's urban environment that were relevant to this study included residential, retail, civic/institutional, private recreation and public parks. For the second set of metrics, we measured the distance from the individual's residential address to the nearest retail, civic/institutional, private recreation facility, public park, as well as public transport access point and food establishment.

\section{Bayesian inference of health outcomes}

Two Bayesian hierarchical models were developed to investigate the relationships between geography and health. The first model elucidates the relationship between geographical exposures and BMI as a continuous variable while controlling for sociodemographic variables. Increased BMI is associated with the higher risk of developing DM, AMI and stroke ${ }^{27-29}$, therefore the second model includes BMI as an additional variable to control for while simultaneously modelling DM, AMI and stroke as binary outcomes, reducing the need to calibrate several models with highly correlated coefficients.

In our first model we let $Y_{i}$ denote the value of individual $i$ 's BMI, and assume

$$
Y_{i} \sim N\left(\mu_{i}, \sigma^{2}\right), \quad Y_{i} \in \boldsymbol{R}^{+}
$$

where the mean is modelled to be a linear combination of predictors,

$$
\mu_{i}=\beta_{0}+\sum_{m=1}^{a} \beta_{m} X_{m, i}
$$

In a model with $a$ explanatory variables with $\beta_{m}$ denoting the coefficients and $X_{m, i}$ the value of the $\mathrm{m}^{\text {th }}$ explanatory variable for the $\mathrm{i}^{\text {th }}$ individual. For the second model, we let $Y_{c, j}$ denote the value of each binary outcome $c$ for each individual $j$,

$$
Y_{c, j} \sim \operatorname{Bern}\left(p_{c, j}\right), \text { for } Y_{c, j} \in\{0,1\}
$$

where $Y_{c, j}$ is drawn from a Bernoulli distribution with probability $p_{c, j}$. In this model, $p_{c, j}$ is defined as 


$$
p_{c, j}=\operatorname{logit}^{-1}\left(\beta_{c}+\sum_{n=1}^{b} \beta_{n} X_{n, j}\right)
$$

with $b$ explanatory variables, $\beta_{n}$ denoting the coefficients for each explanatory variable $n$ and $X_{n, j}$ representing the value for each explanatory variable. Each outcome has a specific intercept $\beta_{c}$, with an individual-specific constant $\sum_{n=1}^{b} \beta_{n} X_{n, j}$ implying identical regression coefficients for DM, AMI and stroke. A more general structure was initially used before settling on a special case with common effect sizes. JAGS, a Gibbs sampler that uses a Markov Chain Monte Carlo algorithm, was used to sample all coefficients from a posterior distribution ${ }^{30}$ in order to calculate $95 \%$ confidence intervals (CIs) for all parameters. Confidence intervals were Agresti-Coull confidence intervals ${ }^{31}$ in order to obtain uncertainty estimates for minizones with $0 \%$ actual or expected prevalence.

\section{Model validation}

Receiver operating characteristic (ROC) curves were used to evaluate overall predictive accuracy for the training and validation datasets. Administratively, Singapore is divided into 55 planning areas with over 200 subzones. From the perspective of identifying at-risk communities, subzones were too coarse, so we further sub-divided the subzones into smaller areas termed "minizones". Each minizone was created to account for property type and was restricted from spanning multiple subzones and electoral boundaries as policies tend to be implemented within political constituency boundaries. The method used to generate these minizones is described in detail in Supplementary information 2. In this way, the map of Singapore was segregated into 954 minizones, of which 662 were populated by at least one individual from the MEC training dataset. Individuals from the validation dataset were intentionally more spatially concentrated, occupying 33 out of 954 minizones. To validate our models, we predicted the mean BMI and the prevalence of individuals with DM, AMI and stroke in each minizone for the CHS validation dataset, excluding minizones with $<10$ people as they would give unstable estimates. These metrics were then evaluated using Hosmer-Lemeshow plots and subsequently visualised. 


\section{National-level risk prediction maps}

Using population estimates from the publicly available 2015 General Household Survey ${ }^{32}$, we generated a synthetic population with the attributes of race, gender and ethnicity. From the same survey, aggregate tables with geographical information on planning area, subzone and dwelling type were used to construct a hill-climbing algorithm that imputed geographical location and dwelling type for the synthetic population. Housing data from the Housing Development Board and property companies was aggregated to construct a full list of dwelling units across Singapore. The synthetic population was then allocated randomly to dwelling units that matched their geographical location and dwelling type. Given the point coordinates of each dwelling unit, we generated geographical exposure variables and estimated house prices for each individual in the population. Smoking status by location was not readily available at a national level, so for the purposes of risk prediction we simulated a bestcase scenario omitting the effects of smoking status on chronic disease risk. With the geographical exposures and synthetic sociodemographic attributes, we predicted mean BMI and average chronic disease risk, denoted by the individual-specific constant $\sum_{n=1}^{b} \beta_{n} X_{n, j}$, by minizone in Singapore. To further investigate the impact of the urban environment on health, we adjusted for all demographic covariates except for house price, due to its dual nature as both a demographic and geographic factor, weighting the respective demographic coefficient with the known distribution of the population from the 2015 General Household Survey, and predicted the mean BMI and chronic disease risk attributable to geographical features.

\section{Software}

All analyses were performed in Microsoft R Open 3.5.2, using JAGS 4.3.0. Spatial analyses and visualisation was done using QGIS 3.6.2 $2^{30,33,34}$. 


\section{$\underline{\text { Results }}$}

Table 1 describes the characteristics of the MEC, our training dataset and the CHS, our validation dataset. Participants in the MEC tended to be younger on average with a mean age of 46 as opposed to the CHS with a mean age of 55 which was expected since the CHS participants came from mature estates. The proportion of males is comparable at $43 \%$ for the MEC and $41 \%$ for the CHS. Oversampling in the MEC also resulted in a larger proportion of Malays and Indians, $26 \%$ and $28 \%$ respectively than the CHS at $13 \%$ and $11 \%$, which follows the national ethnic distribution of Singapore more closely. There were significant differences in the average house price between both datasets. The mature estates that the CHS participants come from predominantly consist of public housing which tends to be lower in value than the mix of private and public housing that MEC participants reside in, evidenced by the mean housing price of $\mathrm{S} \$ 430,000$ for the CHS against $\$ 630,000$ for the MEC. In terms of geography, there were fewer significant differences given the urban density of Singapore. Land use was comparable for both datasets, as well as the distance to the nearest land use type, with the exception of the distances to the nearest private recreation and parks. CHS participants were on average $450 \mathrm{~m}$ from the nearest private recreation outlet compared to the MEC participants at $820 \mathrm{~m}$, almost a difference of twofold. For health outcomes, there were no major differences in the mean and spread of BMI. However, for chronic diseases, CHS participants were slightly unhealthier, likely due to being almost a decade older on average than MEC participants. DM and AMI prevalence of CHS participants were $12 \%$ and $4 \%$ in contrast with the MEC at $10 \%$ and $2 \%$, while stroke prevalence was equal at $1 \%$.

Investigating the relationship between geography and health (Table 2), BMI demonstrates a significant negative association with the distance to the nearest retail outlet, implying that individuals who live closer to retail outlets tend to have increased BMI. None of the geographical covariates were associated with chronic disease risk, although BMI as a risk factor was found to be highly and positively associated with chronic disease risk, suggesting that geography influences health through BMI as a proxy even after adjusting for demographics. Almost all demographic variables displayed 
prominent associations with BMI and chronic disease risk. The direction of the associations was consistent for all of the demographic variables save for gender.

\section{Community and national-level predictions}

To evaluate the predictive ability of the model for DM, AMI and stroke, receiver operating characteristic (ROC) curves were plotted for the MEC (training) and CHS (validation) datasets

(Figure 1). In the training dataset, the area under the curve (AUC) was $>0.8$ for all three conditions. In the CHS, a dataset intentionally chosen for its different demographics, the predictions are accurate with AUCs of $>0.75$ for all three chronic conditions. Geographically, we calculated the expected prevalence of the three chronic conditions along with the expected mean BMI by minizone and compared it to the actual numbers (Figure 2) for each of the 17 minizones represented in the CHS. Root mean square deviation (RMSD) values for each of the 4 outcomes was reasonable, with an RMSD of 0.4 BMI points and $<5.0 \%$ for each of the prevalence estimates. Detailed maps of the actual versus expected BMI and prevalence of the three health outcomes by minizone are also available (Supplementary information 3). The Hosmer-Lemeshow plots in figure 2 also illustrate close correlation between expected and observed quantiles.

Applying the same method to a synthetic population of Singapore and predicting mean BMI and chronic disease risk yields clear clusters of healthy and unhealthy communities (Figure 3). Many of the areas predicted to have increased BMI and higher chronic disease risk correspond to mature estates in Singapore with a large proportion of elderly. In particular, southern and eastern parts of Singapore have higher expected burdens that may warrant intervention. Although demographics is a stronger driver of ill health, geography plays an additional subtler role, with the majority of demography-adjusted relative risks to be within $0.95-1.05$, save for a few outlier areas. Some examples are the southernmost area of Singapore, the island of Sentosa which has a few residential properties for the extremely affluent, and Changi village, the small coastal area at the easternmost point of Singapore. Due to the non-conventional land use in the immediate proximity of those areas, with large amounts of land dedicated to private recreation and almost no other land use type, we observe contrasting effects for BMI and health despite the causal link. Another outlier is the area of 
Lim Chu Kang, the large area in the northwest of Singapore, which is primarily dedicated to agricultural purposes with almost no other land use type, leading to the estimates being mainly driven by demographics. Nevertheless, such outliers are few, with most of our results being consistent. These demography-adjusted maps therefore provide useful information as to the extent that geographic factors impact health through a myriad of means across Singapore, such as proximity to food and access to public and private facilities. 


\section{$\underline{\text { Discussion }}$}

The relationship between geography and health has been established in scientific literature ${ }^{35}$.

Numerous features of the environment, both natural and artificial, have been shown to be associated with health outcomes for instance green spaces have been found to be positively associated with perceived community health ${ }^{36}$ and increased urban mobility with increased physical activity levels ${ }^{37}$. In our study, we found that individuals who lived further away from retail premises were expected to have lower BMI with an expected decrease of -0.95 points per kilometre (95\% CI: [-1.71 to -0.23$])$. One plausible mechanism responsible for this observation is that walking or taking public transport to access retail outlets results in increased physical activity levels with more distant retail outlets requiring more commuting time and therefore more physical activity, as prior research shows that public transport usage was positively associated with physical activity levels through increased walking during commuting ${ }^{38,39}$. This hypothesis is also supported by our reported effect size for the distance to nearest public transport access point on BMI (-1.36, 95\% CI: -2.82 to 0.01$)$, which despite being marginally insignificant, is suggestive that individuals who stay further away from public transport have lower BMI. Despite the inclusion of geographical covariates previously shown to have a positive impact on health such as private recreation facilities and parks in the analysis ${ }^{40,41}$, in our study no significant effect was detected, we hypothesise that park use may be associated with BMI, but that presence of these facilities does not always translate into usage. Another prominent geographical feature associated with health is access to healthy food ${ }^{42}$. In the National Nutrition Survey $2010,61.1 \%$ of participants reported eating at least one meal at hawker centers, locales that serve a variety of affordable but unhealthy food, daily ${ }^{17,43}$.

\section{Identification of high-risk communities}

Applying our model to the population of Singapore, our national-level maps provide useful insights into the potential high-risk communities, which may be useful for evidence-driven policy making ${ }^{35,44}$. From a health services research perspective, maps of chronic disease risk can potentially aid urban planners and regional health systems in ensuring that the facilities in an area are sufficient to support the healthcare needs of the community ${ }^{45}$. From 2009 to 2017, the proportion of those aged 60 and 
above suffering from three or more chronic conditions increased from $19.8 \%$ to $37.0 \%{ }^{46}$, reflecting the growing chronic disease burden and prompting the need for adequate medical care. Urban planners choosing potential locations of homes for the elderly, dialysis centres or other primary care facilities would benefit from information on the spatial distribution of elderly and chronic disease risk. Healthy Urban Planning is one of the themes of the World Health Organisation ${ }^{47}$; the objectives of this theme are to promote healthy lifestyles, facilitate access to healthy food and increase accessibility to healthcare facilities through proper urban planning.

Our method was applied to Singapore but is generalisable to other populations to estimate the geospatial distribution of chronic disease. Globally, there is increased awareness as to the connection between urban planning and the health of communities. A case study of Dortmund, Germany identified significant associations between green space, air quality and socioeconomically disadvantaged communities ${ }^{48}$. In China, a link between population density and obesity was reported based on data from 450 communities over 30 provinces $^{49}$. At the national level, the Canadian Urban Environmental Health Research Consortium was established in 2015 to consolidate a wealth of geospatial exposure data and cohort studies for healthcare research, including features such as transportation networks and land use ${ }^{50}$. With the appropriate combination of census and geospatial information, geospatial distributions of disease similar to ours can be generated to better inform policy at the city scale that many planners operate in.

\section{Limitations}

A major limitation of our study is the cross-sectional study design. Although based off cohort studies, our observations were derived from a cross-section of geographical features, so aetiological conclusions cannot be drawn. Differential usage of retail, recreational and food facilities was also not explored, due to constraints on collecting such detailed information on a large scale. Quantifying the usage of facilities could potentially improve model accuracy in predicting health outcomes, as lifestyle choices such as diet and exercise heavily influence chronic disease risk ${ }^{13,51}$. The land use category of retail is diverse and can positively or negatively affect health, illustrated by the distribution of healthy food outlets in Australia ${ }^{52}$ and fried chicken stores in South Korea ${ }^{53}$. Potential 
improvements to this study would be obtaining the actual anonymised geographic distribution of the population with detailed health information as opposed to a synthetically reconstructed population for additional validation, although this was not possible due to issues of data privacy.

\section{$\underline{\text { Conclusion }}$}

Geographical distributions of chronic disease risk are instrumental in understanding differences in community health at a national level and identifying high-risk communities. Understanding the joint role that demography and geography plays in impacting the health of communities helps in constructing predictive models of health, which are critical for evidence-based policy-making. Healthy Urban Planning initiatives would be able to enhance decision-making using maps of chronic disease risk in order to improve population health through urban design. At a population level, urban interventions allowing for greater access to healthcare and healthier lifestyle options have a small effect across the whole population but may yet prove to be efficacious as urbanisation increases globally.

\section{$\underline{\text { Declarations }}$}

\section{Ethics approval and consent to participate}

This study was approved by the NUS-IRB, reference number S-19-121.

\section{Consent for publication}

The authors hereby consent to publication of the article.

\section{Availability of data and materials}

The data that support the findings of this study are available from the Saw Swee Hock School of Public Health but restrictions apply to the availability of these data, which were used under license for the current study, and so are not publicly available. Data can however be requested from the following website https://blog.nus.edu.sg/sphs/. 


\section{Competing interests}

The authors declare no competing interests.

\section{Funding}

The work is supported by the National Research Foundation's Virtual Singapore grant R-608-000197-281 and the National Medical Research Council's Singapore Population Health Improvement Centre.

\section{Author's contributions}

KWT performed the literature search, data analysis and produced the figures for the manuscript and was a major contributor in writing the manuscript. QY aided in the data analysis and contributed to writing the manuscript. YAL and JRK performed data collection for the study. ARC participated in the study design and was the principal investigator of the study. BLD also participated in the study design and was a major contributor in writing the manuscript.

\section{Acknowledgements}

Not applicable. 


\section{$\underline{\text { References }}$}

1. Cheah, J. Chronic disease management: a Singapore perspective. BMJ 323, 990-993 (2001).

2. Chapel, J. M., Ritchey, M. D., Zhang, D. \& Wang, G. Prevalence and Medical Costs of Chronic Diseases Among Adult Medicaid Beneficiaries. Am. J. Prev. Med. 53, S143-S154 (2017).

3. Cost of managing complications resulting from type 2 diabetes mellitus in Canada. Available at: https://www.ncbi.nlm.nih.gov/pmc/articles/PMC153533/. (Accessed: 19th September 2019)

4. Shibayama, T., Noguchi, H., Takahashi, H. \& Tamiya, N. Relationship between social engagement and diabetes incidence in a middle-aged population: Results from a longitudinal nationwide survey in Japan. J. Diabetes Investig. 9, 1060-1066 (2018).

5. Fu, S., Huang, N. \& Chou, Y.-J. Trends in the Prevalence of Multiple Chronic Conditions in Taiwan From 2000 to 2010: A Population-Based Study. Prev. Chronic. Dis. 11, (2014).

6. Kaur, P., Saxena, N. \& Zhu, Z. Effect of Asian BMI on risk of chronic disease progression: A Singapore perspective. Proc. Singap. Healthc. 27, 270-276 (2018).

7. Ministry of Health, Singapore. Executive Summary on National Population Health Survey 2016/17. (2017).

8. Health Promotion Board, Singapore. Singapore Myocardial Infarction Registry Annual Report 2016. (2016).

9. Health Promotion Board, Singapore. Singapore Stroke Registry Annual Report 2016. (2016).

10. Chia, D. B., Wong, L. Y., Liu, D. Y. K. \& Toh, M. P. H. S. Predictive factors of developing type 2 diabetes mellitus, Acute Myocardial Infarction and stroke in a cohort with Impaired Fasting Glucose in Singapore. Diabetes Res. Clin. Pract. 132, 59-67 (2017).

11. Phan, T. P. et al. Forecasting the burden of type 2 diabetes in Singapore using a demographic epidemiological model of Singapore. BMJ Open Diabetes Res. Care 2, e000012 (2014).

12. Lee, H. et al. Influence of urban neighbourhood environment on physical activity and obesityrelated diseases. Public Health 129, 1204-1210 (2015). 
13. Willett, W. C. et al. Prevention of Chronic Disease by Means of Diet and Lifestyle Changes. in Disease Control Priorities in Developing Countries (eds. Jamison, D. T. et al.) (World Bank, 2006).

14. Boone-Heinonen, J. et al. The neighborhood energy balance equation: does neighborhood food retail environment + physical activity environment = obesity? The CARDIA study. PloS One 8 , e85141 (2013).

15. Kelli, H. M. et al. Living in Food Deserts and Adverse Cardiovascular Outcomes in Patients With Cardiovascular Disease. J. Am. Heart Assoc. Cardiovasc. Cerebrovasc. Dis. 8, (2019).

16. Hamano, T., Kawakami, N., Li, X. \& Sundquist, K. Neighbourhood Environment and Stroke: A Follow-Up Study in Sweden. PLOS ONE 8, e56680 (2013).

17. Naidoo, N. et al. Determinants of eating at local and western fast-food venues in an urban Asian population: a mixed methods approach. Int. J. Behav. Nutr. Phys. Act. 14, (2017).

18. Ministry of Health, Singapore. Chronic disease management program. (2018).

19. Tan, K. H. X. et al. Cohort Profile: The Singapore Multi-Ethnic Cohort (MEC) study. Int. J. Epidemiol. (2018).

20. Yeo, K. K. et al. Ethnicity modifies the association between diabetes mellitus and ischaemic heart disease in Chinese, Malays and Asian Indians living in Singapore. Diabetologia 49, 2866-2873 (2006).

21. Low, L. L. et al. Housing as a Social Determinant of Health in Singapore and Its Association with Readmission Risk and Increased Utilization of Hospital Services. Front. Public Health 4, (2016).

22. Wang, X. et al. The Association between Socioeconomic Status, Smoking, and Chronic Disease in Inner Mongolia in Northern China. Int. J. Environ. Res. Public. Health 16, (2019).

23. Owen, N. et al. Associations of neighborhood environmental attributes with adults' objectivelyassessed sedentary time: IPEN adult multi-country study. Prev. Med. 115, 126-133 (2018).

24. Adams, M., Chapman, J., Sallis, J., Frank, L. \& International Physical Activity and Environment Network Study Coordinating Center. Built environment and physical activity: GIS templates and variable naming conventions. 107 (2012). 
25. Oliver, L. N., Schuurman, N. \& Hall, A. W. Comparing circular and network buffers to examine the influence of land use on walking for leisure and errands. Int. J. Health Geogr. 6, 41 (2007).

26. Urban Redevelopment Authority, Singapore. Urban Redevelopment Authority Master Plan 2014. (2014).

27. Al-Goblan, A. S., Al-Alfi, M. A. \& Khan, M. Z. Mechanism linking diabetes mellitus and obesity. Diabetes Metab. Syndr. Obes. Targets Ther. 7, 587-591 (2014).

28. Tun, N. N., Arunagirinathan, G., Munshi, S. K. \& Pappachan, J. M. Diabetes mellitus and stroke: A clinical update. World J. Diabetes 8, 235-248 (2017).

29. Tavani, A., Bertuzzi, M., Gallus, S., Negri, E. \& La Vecchia, C. Diabetes mellitus as a contributor to the risk of acute myocardial infarction. J. Clin. Epidemiol. 55, 1082-1087 (2002).

30. Plummer, M. JAGS: A Program for Analysis of Bayesian Graphical Models Using Gibbs Sampling. 10 (2003).

31. Agresti, A. \& Coull, B. A. Approximate is Better than "Exact" for Interval Estimation of Binomial Proportions. Am. Stat. 52, 119-126 (1998).

32. Department of Statistics, Singapore. General Household Survey 2015. (2016).

33. Microsoft \& R Core Team. Microsoft R Open. (2019).

34. QGIS Development Team (2019) \& Open Source Geospatial Foundation Project. QGIS Geographic Information System. (2019).

35. Dummer, T. J. B. Health geography: supporting public health policy and planning. CMAJ Can. Med. Assoc. J. 178, 1177-1180 (2008).

36. Maas, J., Verheij, R. A., Groenewegen, P. P., de Vries, S. \& Spreeuwenberg, P. Green space, urbanity, and health: how strong is the relation? J. Epidemiol. Community Health 60, 587-592 (2006).

37. Stevenson, M. et al. Land-use, transport and population health: estimating the health benefits of compact cities. Lancet Lond. Engl. 388, 2925-2935 (2016). 
38. Rissel, C., Curac, N., Greenaway, M. \& Bauman, A. Physical Activity Associated with Public Transport Use-A Review and Modelling of Potential Benefits. Int. J. Environ. Res. Public. Health 9, 2454-2478 (2012).

39. Saelens, B. E., Vernez Moudon, A., Kang, B., Hurvitz, P. M. \& Zhou, C. Relation Between Higher Physical Activity and Public Transit Use. Am. J. Public Health 104, 854-859 (2014).

40. Higgerson, J., Halliday, E., Ortiz-Nunez, A., Brown, R. \& Barr, B. Impact of free access to leisure facilities and community outreach on inequalities in physical activity: a quasi-experimental study. J. Epidemiol. Community Health 72, 252-258 (2018).

41. Müller-Riemenschneider, F. et al. Prescribing Physical Activity in Parks to Improve Health and Wellbeing: Protocol of the Park Prescription Randomized Controlled Trial. Int. J. Environ. Res. Public. Health 15, (2018).

42. Rose, D. Access to Healthy Food: A Key Focus for Research on Domestic Food Insecurity. J. Nutr. 140, 1167-1169 (2010).

43. Health Promotion Board, Singapore. National Nutrition Survey. (2010).

44. Brownson, R. C., Chriqui, J. F. \& Stamatakis, K. A. Understanding Evidence-Based Public Health Policy. Am. J. Public Health 99, 1576-1583 (2009).

45. Musa, G. J. et al. Use of GIS Mapping as a Public Health Tool-From Cholera to Cancer. Health Serv. Insights 6, 111-116 (2013).

46. The Straits Times. Proportion of older adults with multiple chronic diseases surges. (2019).

47. Barton, H. \& Grant, M. Urban Planning for Healthy Cities. J. Urban Health Bull. N. Y. Acad. Med. 90, 129-141 (2013).

48. Flacke, J., Schüle, S. A., Köckler, H. \& Bolte, G. Mapping Environmental Inequalities Relevant for Health for Informing Urban Planning Interventions-A Case Study in the City of Dortmund, Germany. Int. J. Environ. Res. Public. Health 13, (2016). 
49. Wang, R., Feng, Z., Xue, D., Liu, Y. \& Wu, R. Exploring the links between population density, lifestyle, and being overweight: secondary data analyses of middle-aged and older Chinese adults. Health Qual. Life Outcomes 17, (2019).

50. Brook, J. R. et al. The Canadian Urban Environmental Health Research Consortium - a protocol for building a national environmental exposure data platform for integrated analyses of urban form and health. BMC Public Health 18, 114 (2018).

51. Booth, F. W., Roberts, C. K. \& Laye, M. J. Lack of exercise is a major cause of chronic diseases. Compr. Physiol. 2, 1143-1211 (2012).

52. Moayyed, H., Kelly, B., Feng, X. \& Flood, V. Is Living near Healthier Food Stores Associated with Better Food Intake in Regional Australia? Int. J. Environ. Res. Public. Health 14, (2017).

53. Kim, J., Shon, C. \& Yi, S. The Relationship between Obesity and Urban Environment in Seoul. Int. J. Environ. Res. Public. Health 14, (2017). 
Figures
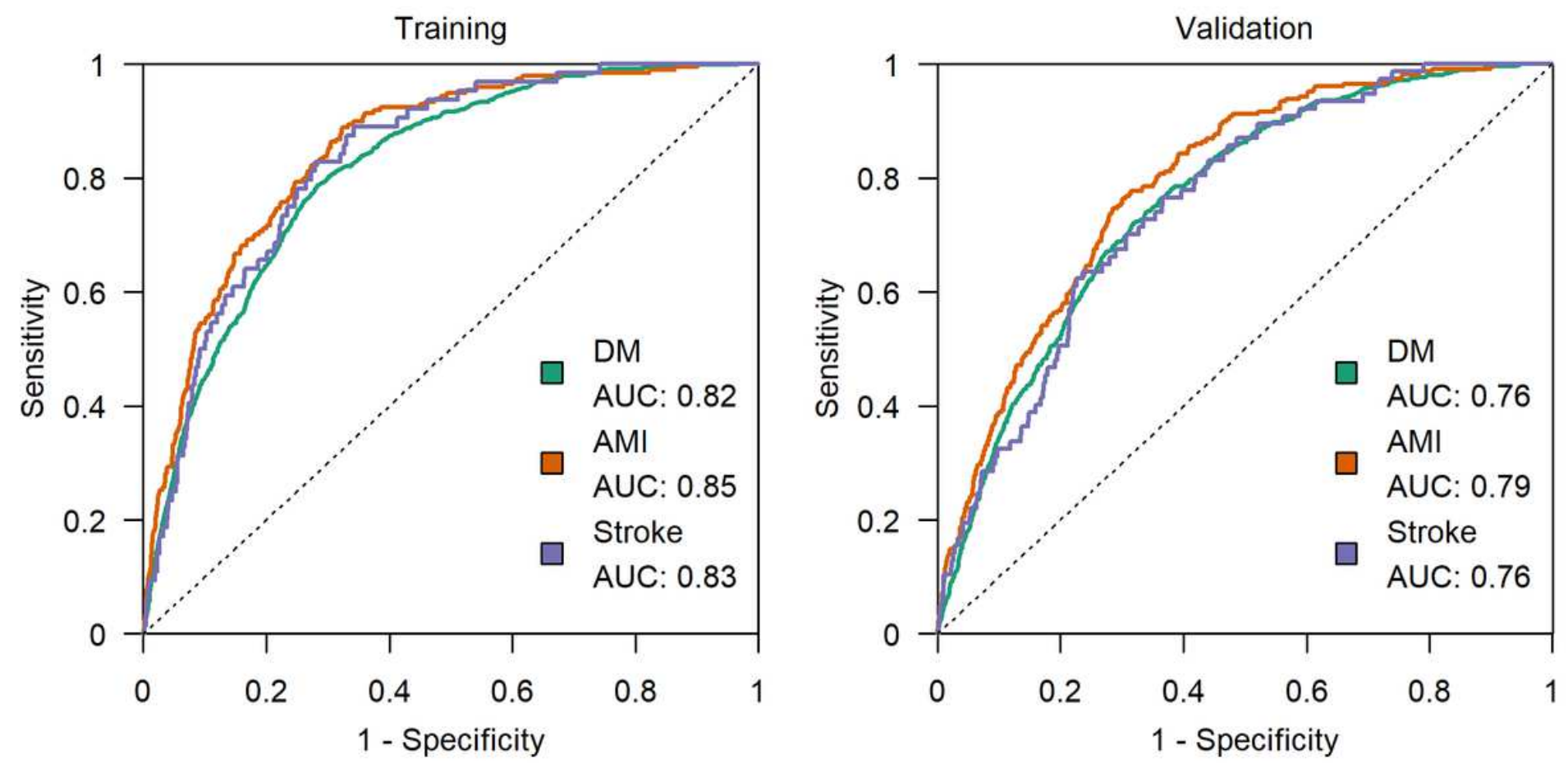

Figure 1

ROC curves of the Multi-Ethnic Cohort (training) and the Community Health Study (validation) for DM, $\mathrm{AMl}$ and stroke. 

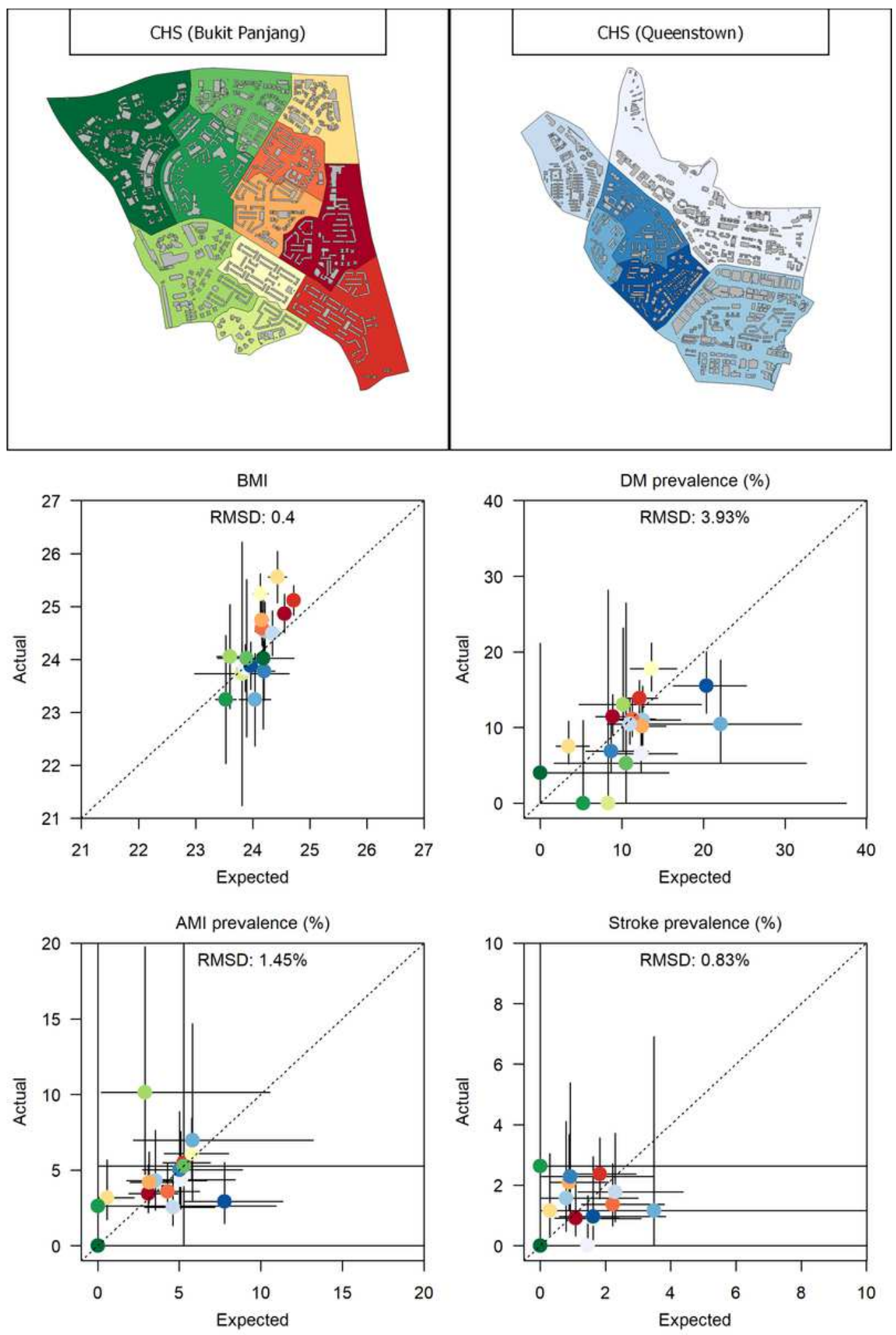

\section{Figure 2}

Maps of the 17 minizones in Bukit Panjang and Queenstown with more than 10 residents and HosmerLemeshow plots of mean BMI, DM, AMI and stroke prevalence. Confidence intervals are Agresti-Coull confidence intervals. Colours of the minizones on the maps correspond to the colours of the respective points in the Hosmer-Lemeshow plots. Some confidence intervals have been truncated. 


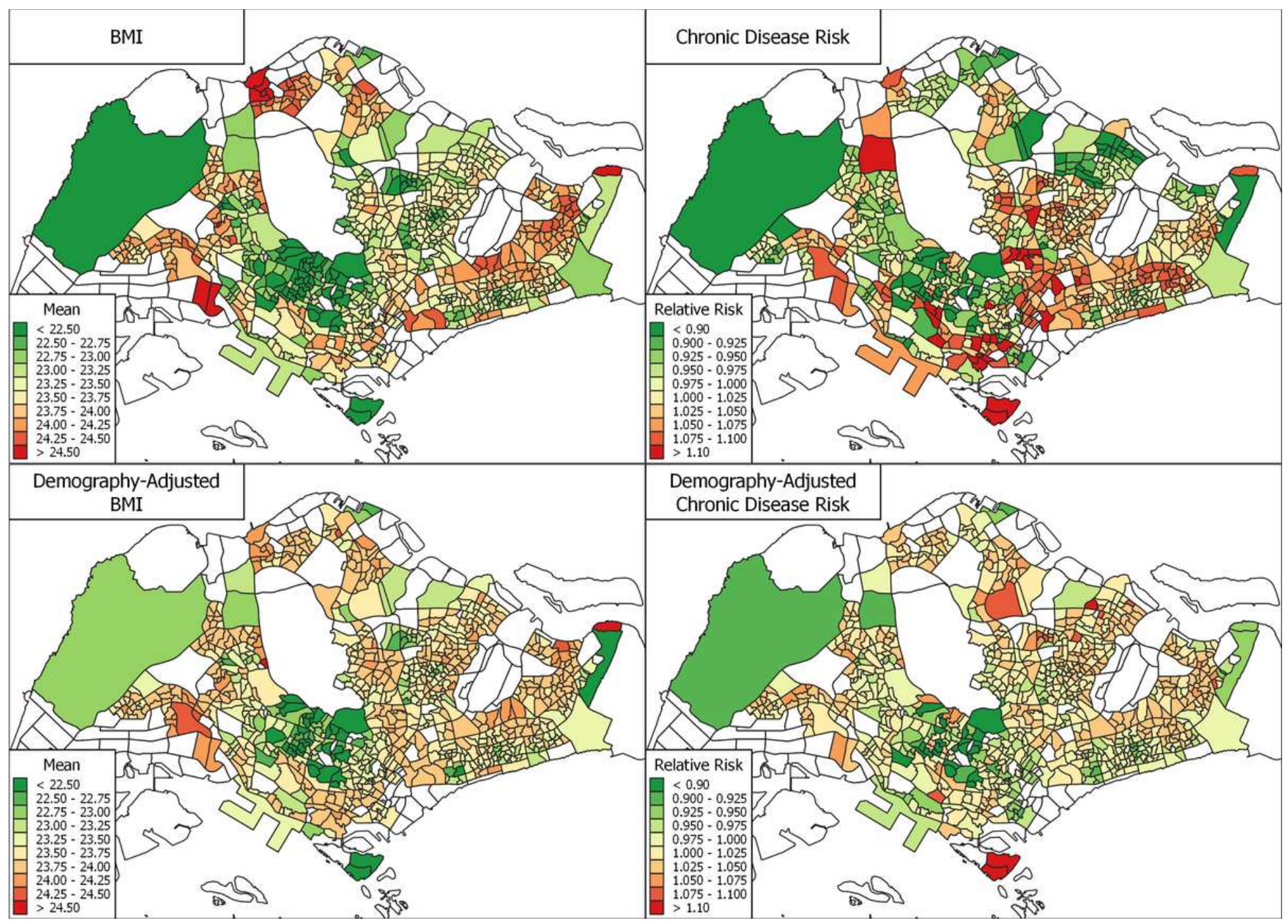

Figure 3

National-level maps of mean BMI, relative risk of chronic disease, demography-adjusted mean BMI and demography-adjusted chronic disease risk. Red areas have worse health outcomes and green areas have better health outcomes. Areas with less than 100 residents were excluded and are coloured white. 